\title{
Characteristics of Smell Identification Test in Patients With Parkinson Disease
}

\author{
Hisami Fujio ${ }^{1}$ (1) · Go Inokuchi ${ }^{1} \cdot$ Shun Tatehara ${ }^{1} \cdot$ Shunsuke Kuroki ${ }^{1} \cdot$ Yuriko Fukuda $^{1} \cdot$ Hisamoto Kowa $^{2} \cdot$ Ken-ichi Nibu $^{1}$ (i) \\ ${ }^{1}$ Department of Otolaryngology-Head and Neck Surgery, Kobe University Graduate School of Medicine, Kobe; \\ ${ }^{2}$ Department of Rehabilitation Science, Kobe University Graduate School of Health Sciences, Kobe, Japan
}

Objectives. Parkinson disease (PD) is frequently associated with olfactory disorder at early stage, which is caused by deposition of Lewy bodies emerging from the olfactory bulb to higher olfactory centers. Early detection of olfactory disorder in the patients with PD may lead to the early diagnosis and treatment for this refractory disease.

Methods. Visual analog scale (VAS), Jet Stream Olfactometry, and Japanese smell identification test, Open Essence (OE), were carried out on 39 patients with PD. Thirty-one patients with postviral olfactory disorder (PVOD), which was caused by the olfactory mucosal dysfunction, were also enrolled in this study as control.

Results. There were no significant differences in detection thresholds (2.2 vs. 1.4, $P=0.13$ ), recognition thresholds (3.9 vs. $3.5, P=0.39)$ and $\mathrm{OE}(4.8$ vs. $4.2, P=0.47)$ between PVOD and PD, while VAS scores of PVOD and PD were significantly different $(2.0$ and $6.2, P<0.01)$. In $\mathrm{OE}$, significant differences were observed in the accuracy rates of menthol ( $68 \%$ vs. $44 \%, P=0.04)$ and Indian ink ( $42 \%$ vs. $15 \%, P=0.01)$ between PVOD and PD. Of particular interest, patients with PVOD tended to select "no detectable," while patients with PD tended to select wrong alternative other than "no smell detected."

Conclusion. Discrepancy between VAS and OE, and high selected rates of wrong alternative other than "undetectable" in $\mathrm{OE}$ might be significant signs of olfactory dysfunction associated with PD.

Keywords. Postviral Olfactory Disorder; Parkinson Disease; Open Essence; Smell Identification Test

\section{INTRODUCTION}

Parkinson disease is one of the most common central disorder caused by Lewy bodies emerge from the olfactory bulb to a higher olfactory center [1] and has been known to be frequently associated with reported olfactory dysfunction [2,3], as reported by Doty using University of Pennsylvania Smell Identification Test (UPSIT) in PD [4]. Since olfactory dysfunction manifests at early stage of PD, examination of olfaction has been expected to lead to early diagnosis and initiation of treatment of this refrac-

\footnotetext{
- Received July 28, 2018

Revised October 17, 2018

Accepted November 1, 2018

- Corresponding author: Ken-ichi Nibu

Department of Otolaryngology-Head and Neck Surgery, Kobe University

Graduate School of Medicine, 7-5-2 Kusunoki-cho, Chuo-ku,

Kobe 650-0017, Japan

Tel: +81-78-382-6020, Fax: +81-78-382-6039

E-mail: nibu@med.kobe-u.ac.jp
}

tory disease [5]. However, patients with PD mostly do not become aware of their olfactory disturbance until PD advances to show it typical motor symptom [6]. Besides, in daily clinical practice, we often encounter elderly patients with olfactory disorders due to various causes including aging, smoking, infection, medication other than PD [7].Thus, it is hard to differentiate olfactory dysfunction due to PD from olfactory dysfunction due to other etiologies in daily practice.

To date, several smell identification tests have been developed to evaluate olfaction. UPSIT [8] and the Cross-Cultural Smell Identification Test [9] have been introduced in the United States and the "Sniffing' sticks" test has been used in Germany [10]. However, several odors included in these tests are unfamiliar to the Japanese. Open Essence (OE) was developed for Japanese by the National Institute of Advanced Industrial Science and Technology, and has been marketed since September 2008. Like UPSIT, OE presents 12 odors, all of which have been confirmed as familiar to the healthy Japanese with normal olfaction and

Copyright @ 2019 by Korean Society of Otorhinolaryngology-Head and Neck Surgery.

This is an open-access article distributed under the terms of the Creative Commons Attribution Non-Commercial License (http://creativecommons.org/licenses/by-nc/4.0)

which permits unrestricted non-commercial use, distribution, and reproduction in any medium, provided the original work is properly cited. 
has been validated as simple and useful examination tool for olfaction [11].

In this study, to evaluate the usefulness of smell identification test in order to diagnose Parkinson disease among the patients with olfactory disorders, we examined the olfaction of patients with postviral olfactory disorder (PVOD) and patients with PD using OE.

\section{MATERIALS AND METHODS}

\section{Subjects}

During the period between May 2013 to November 2014, olfaction was assessed with visual analog scale (VAS), Jet Stream Olfactometry (JSO), and OE on 31 patients with PVOD and 39 patients with PD at the Department of Otolaryngology-Head and Neck Surgery, Kobe University Hospital. Of the 31 patients with PVOD, five were men and 26 were women with a mean age of 62.6 years old ranging from 37 to 85 years. Of the 39 patients with PD, 22 were men and 17 were women with a mean age of 67.5 years old ranging from 45 to 86 . All of them were diagnosed at the Department of Neurology, Kobe University Hospital and had sign of olfactory disfunction. All the experiments in this study were approved by the Ethical Committee of Kobe University Graduate School of Medicine (No. 1539). Written informed consents were obtained from all subjects prior to study participation.

\section{Visual analog scale}

In the present study, we used VAS to measure the symptoms of olfactory dysfunction. VAS consists of a 10-cm line, both ends of which have statements of the maximal and minimal extremes. The subjects were asked to indicate their feelings by marking the line at the appropriate point between the two extreme statements, defined as "anosmia" and "normal [12]."

\section{Jet stream olfactometry}

In JSO, one of the five sources of odor, namely, A (beta-phenyl ethyl alcohol), B (methyl cyclopentenone), C (isovaleric acid), D (gamma-undecalactone), and $\mathrm{E}$ (skatole) were placed into each bottle, which was then mounted on the stimulation compressor to send odor-containing air directly into the nasal cavity of each

\section{H I G G H L I G G H T S}

- Olfaction of patients with Parkinson disease was evaluated by smell identification test (Open Essence).

- Discrepancy between visual analog test and Open Essence was found in patients with Parkinson disease.

- Patients with Parkinson disease tended to select wrong alternatives in Open Essence. subject for measurement [13]. The nozzle was inserted through the nostril of each subject toward the olfactory cleft to give an odor stimulus during inspiration. All odorants were presented in eight 10 -fold serial log step dilutions (from -2 to 5 by 1 ), with the exception of odorant $\mathrm{B}$, which has only seven dilution steps (from -2 to 4 by 1 ). Recognition threshold was defined as the lowest dilution step at which the odor can be correctly identified. The recognition thresholds for five odorants are averaged, and this value is used to assign a diagnostic category representing the level of olfactory function as follows: scores are normal (-2 to 1 ), mild hyposmia (1.1 to 2.5$)$, moderate hyposmia (2.6 to 4.0 ), severe hyposmia (4.1 to 5.5), and anosmia (5.6 or more). JSO was applied to each nostril. Lower threshold was determined as the threshold of the patient.

\section{Card kit for assessment of olfactory identification test (OE)}

$\mathrm{OE}$ is a card-type test kit developed in Japan for assessment of olfactory identification abilities [13]. Micro-capsulated fragrance was printed on one side of the twice-folded card, and the other side carries information on alternatives. When the subject opens the twice-folded card, micro-capsule breaks and odor is presented. There are 12 kinds of card (A though $\mathrm{L}$ ) carrying different odors: (A) India ink, (B) lumber, (C) perfume, (D) menthol, (E) Japanese orange, (F) curry, $(\mathrm{G})$ gas (for household use), (H) rose, (I) Japanese cypress, (J) odor of stocking stinking with sweat, (K) condensed milk, and $(\mathrm{L})$ fried garlic. All of these odors are familiar to Japanese people [10]. The subject was asked to select the type of odor carried on a given card from the alternatives printed on the right side of the card and to enter the answer into the answer sheet. The six alternatives were composed of "correct odor," "odor closest to the correct odor," "odor close to the correct odor," "odor far different from the correct odor," "detectable but not recognizable (unidentifiable)," and "no smell detected (undetectable)" in accordance with cluster analysis. Each correct answer was given 1 point and each wrong answer was given 0 point. The points for all cards were totaled. Selecting the alternative "unidentifiable" or "undetectable" was also counted as a wrong answer. Reported normal range of $\mathrm{OE}$ has been reported as above 8 [11].

\section{Mini-mental state examination}

Cognitive impairment of the patients with PD was evaluated using mini-mental state examination (MMSE). Twenty-eight to 30 points were defined as normal, 24 to 27 points were defined as mild cognitive impairment and 23 points or less were defined as dementia.

\section{Statistical analysis}

On the basis of the data from these tests, statistical analyses were performed using $t$-test, chi-square test or Spearman rank correlation coefficient as required with Statcel 2 (OMS Publishing Inc., Tokorozawa, Japan). A $P$-value $<0.05$ was considered as significant. 


\section{RESULTS}

No significant differences were observed regarding age, average detection threshold, average recognition threshold and the number of correct answers of OE between PVOD and PD groups (Table 1). Also, no significant difference was observed in the difference between recognition average value and detection average value between PVOD and PD groups, although difference was larger in PD group (2.10 vs. 1.62, $P=0.13$ ). Instead, significant difference was observed in VAS between PVOD and PD groups (2.0 vs. $6.2, P<0.01)$ (Table 1$)$.

Correct answer rates of the 12 odors are shown in Table 2. In PVOD group, correct answer rate was highest in curry $(71 \%)$, followed by menthol $(68 \%)$ and odor of stocking with sweat (52\%). In contrast, no significant difference was observed in the correct answer rate among the 12 odors in PD group. Between PVOD and PD groups, correct answer rates of menthol and Indian ink were significantly different ( $68 \%$ vs. $44 \%, P=0.04 ; 42 \%$

Table 1. Results of visual analog scale, jet stream olfactometer and Open Essence in PVOD and PD groups

\begin{tabular}{lccc}
\hline Variable & PVOD & PD & $P$-value \\
\hline No. of patients (male:female) & $31(5: 26)$ & $39(22: 17)$ & $<0.01$ \\
Mean age (yr) & 62.6 & 67.5 & 0.068 \\
Visual analog scale & 2.0 & 6.2 & $<0.01$ \\
Detection thereshold & 2.2 & 1.4 & 0.133 \\
Recognition threshold & 3.9 & 3.5 & 0.392 \\
Open essence & 4.8 & 4.2 & 0.469 \\
\hline
\end{tabular}

PVOD, postviral olfactory disorder; PD, Parkinson disease. vs. $15 \%, P=0.01)$.

Of particular interest, patients with PD clearly tended to select wrong alternatives other than "undetectable" in OE compared with patients with PVOD group as shown in Fig. 1. The rates of "undetectable" in the wrong answers of $\mathrm{OE}$ (the number of selected "undetectable" divided by the number of selected wrong answers) were significantly different excepting curry, Japanese cypress and condensed milk between PVOD group and PD group (Table 3). Fig. 2 shows the distribution of the selected rates of alternatives other than "undetectable" in the wrong answers according to the number of correct answers in OE. All the patients with PD, except one patient who selected correct answers in all odors, selected $70 \%$ or more of alternatives other than "undetectable" as wrong answers.

Table 2. Correct answer rates of 12 odors

\begin{tabular}{lccc}
\hline Variable & PVOD (\%) & PD (\%) & $P$-value \\
\hline Curry & 71 & 54 & 0.144 \\
Menthol & 68 & 44 & 0.044 \\
Stocking with sweat & 52 & 51 & 0.987 \\
Japanese cypress & 52 & 41 & 0.377 \\
Condensed milk & 48 & 33 & 0.202 \\
Indian ink & 42 & 15 & 0.013 \\
Japanese orange & 39 & 39 & 0.983 \\
Gas for household use & 29 & 28 & 0.939 \\
Perfume & 23 & 36 & 0.227 \\
Lumber & 19 & 28 & 0.391 \\
Fried garlic & 19 & 33 & 0.191 \\
Rose & 16 & 21 & 0.639 \\
\hline
\end{tabular}

PVOD, postviral olfactory disorder; PD, Parkinson disease.
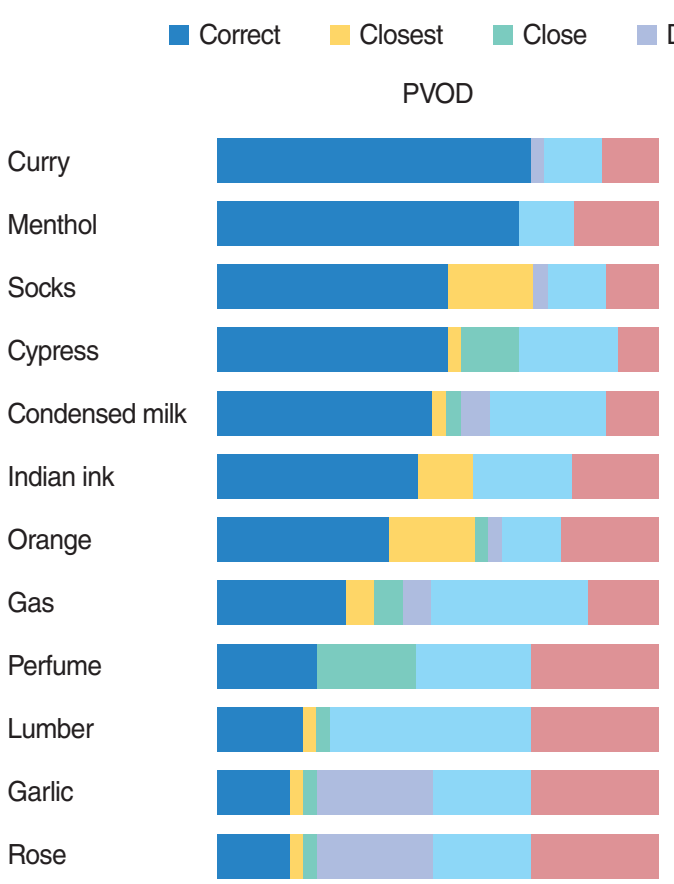

Different Unidentifiable $\square$ Undetectable

PD

$P$-value

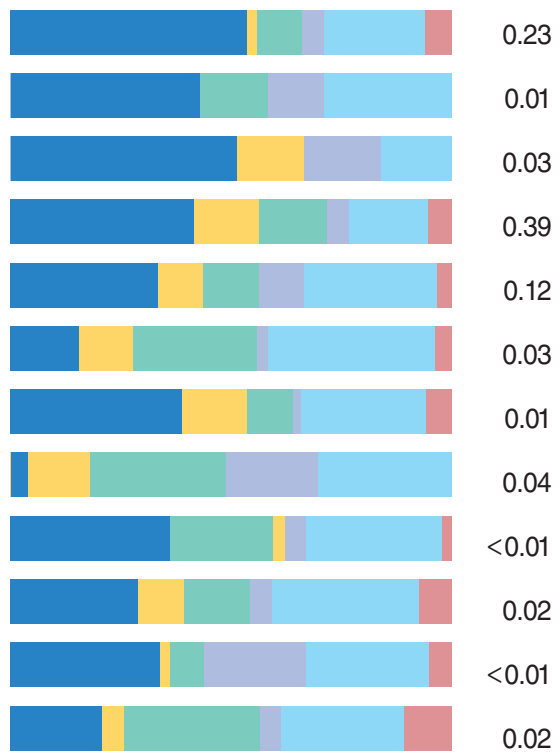

Fig. 1. Selected answers of 12 odors in PVOD and PD groups. PVOD, postviral olfactory disorder; PD, Parkinson disease. 
Table 3. Selected rates of undetectable in the wrong answers

\begin{tabular}{lccc}
\hline Variable & PVOD (\%) & PD (\%) & $P$-value \\
\hline Rose & 54.1 & 13 & 0.001 \\
Curry & 44.4 & 11 & 0.073 \\
Menthol & 40 & 0 & $<0.001$ \\
Perfume & 37.5 & 4 & 0.011 \\
Japanese orange & 36.9 & 8.3 & 0.028 \\
Lumber & 36 & 10.8 & 0.030 \\
Indian ink & 35.3 & 3 & 0.004 \\
Fried garlic & 34.7 & 3.8 & 0.019 \\
Stocking with sweat & 27.7 & 0 & 0.029 \\
Condensed milk & 25 & 3.8 & 0.011 \\
Gas for household use & 22.8 & 0 & 0.012 \\
Japanese cypress & 20 & 9 & 0.298 \\
\hline
\end{tabular}

PVOD, postviral olfactory disorder; PD, Parkinson disease.

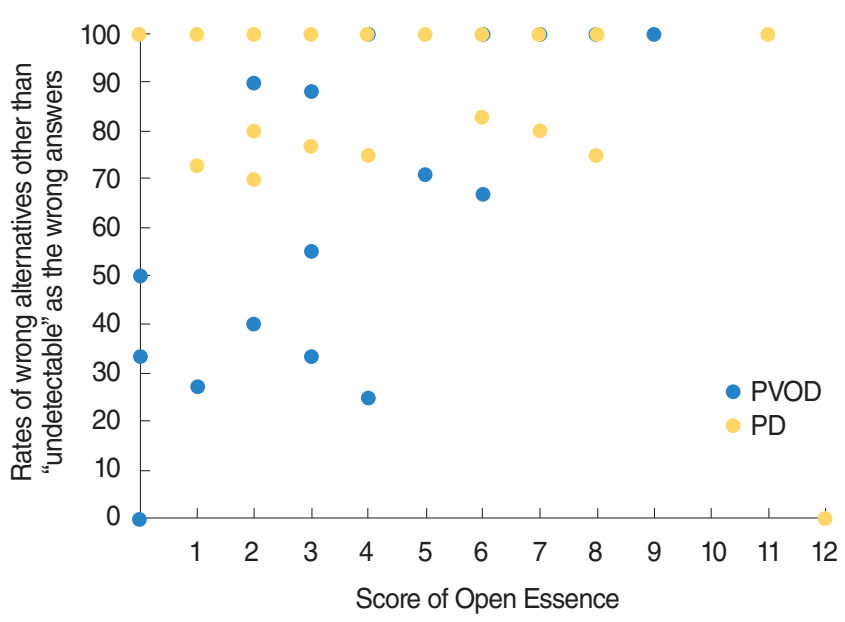

Fig. 2. Distribution of the selected rates of wrong alternatives other than "undetectable" as the wrong answers according to the number of correct answers in Open Essence. PVOD, postviral olfactory disorder; PD, Parkinson disease.

To evaluate the usefulness of $\mathrm{OE}$ as the screening tool for Parkinson disease, we performed the receiver operating characteristic curve analysis. Maximum area under the curve $(0.657)$ was obtained when cutoff value was set up at $77 \%$ in the selected rates of alternatives other than "undetectable" in the wrong answers. The sensitivity and specificity were 0.909 and 0.5 , respectively (Fig. 3).

Number of correct answers of OE (OE scores) according to MMSE scores were demonstrated in Fig. 4. By statistical analysis using Spearman rank correlation coefficient, no significant correlation was observed between MMSE scores and number of correct answers of OE.

\section{DISCUSSION}

Doty [4] studied the characteristics of olfactory dysfunction of

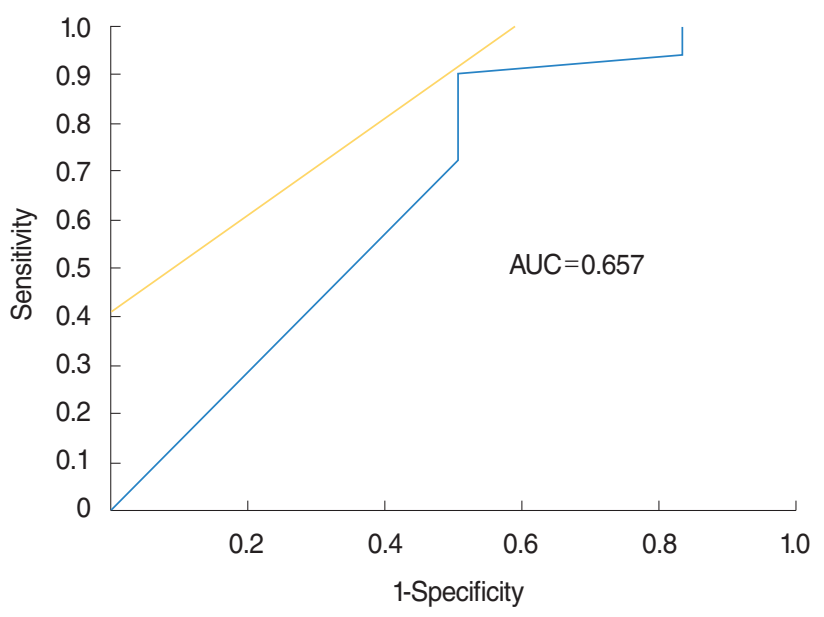

Fig. 3. Receiver operating characteristic curve between postviral olfactory disorder (PVOD) and Parkinson disease (PD) groups. When cutoff value was set up at $77 \%$ in the selected rates of wrong answers other than "undetectable," the sensitivity was 0.909; specificity was 0.5 ; and area under the curve (AUC) was 0.657 .

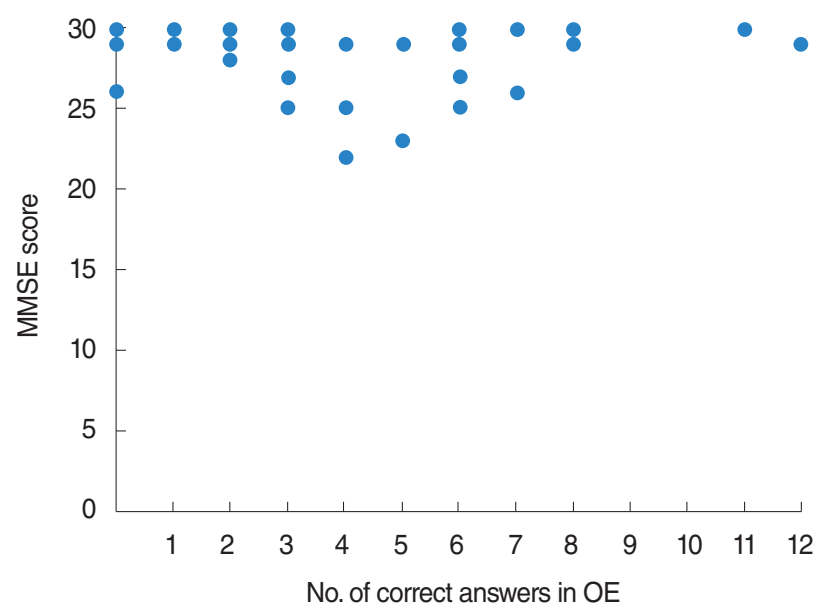

Fig. 4. Number of correct answers of Open Essence (OE) scores according to mini-mental state examination (MMSE) scores. No significant correlation was observed between MMSE and OE scores.

the patients with PD and reported detection and identification dysfunction in approximately $75 \%$ and $90 \%$ of the patients with PD. In PD, a central olfactory disorder caused by Lewy bodies emerges from the olfactory bulb to a higher olfactory center during the premotor phase [1], suggesting olfactory impairment may occur in early stages of this disease [2,3]. Accordingly, in longitudinal studies, hyposmia was associated with a significantly increased risk to develop PD [5] and presence of olfactory impairment at baseline predicted the emergence of dementia and focal changes in brain structures [6]. Thus, olfactory assessment may be useful in early diagnosis and treatment of PD. However, it is well known that olfaction is deteriorating with age due to various etiologies as mentioned above [7].Thus, development of simple diagnostic tool to differentiate PD from 
olfactory dysfunction due to other etiologies emergent.

Since PD-associated olfactory dysfunction has been reported to involve several domains of odor perception, i.e., detection, identification (discrimination), and memory which are likely related to olfactory bulbs, olfactory tract and olfactory coretexes [14], we included patients with PVOD caused by a direct damage of the olfactory receptor cells $[15,16]$ as control group and evaluate various domains of odor perception.

In the JSO evaluation, no significant difference was observed in recognition average value and detection average value between PVOD and PD groups although the difference between recognition threshold and detection threshold was larger in PD group. Instead, significant difference was observed in the VAS scores between PVOD and PD groups, indicating that patients with PD mostly do not become aware of their olfactory disturbance as previously reported. Most possible explanation for this finding is that hyposmia is progressing gradually and is not complete in the patients with PD [4,17].

Since the olfaction was mainly disturbed in olfactory epithelium in PVOD $[15,16]$, but in olfactory bulb and upper olfactory cortexes which are mainly responsible for odor recognition and discrimination in PD [1], we expected that olfactory identification test might be helpful to differentiate PD from PVOD. However, no significant difference was observed in the total scores of $\mathrm{OE}$ between PVOD and PD groups in the present study. While total score of VAS by itself was not useful for differential diagnosis, these results suggested that discrepancy between VAS scores and scores of JCO and/or OE might be a characteristic sign of PD.

By close analysis, correct answer rates of menthol and Indian ink were significantly different. Since correct answer rate of menthol but not Indian ink also decreased in the olfactory disturbance due to aging as we previously reported [18], wrong answer in Indian ink but not in menthol might be a useful sign of PD. Neurodegenerative changes are commonly observed in the regions of the brain responsible for olfactory perception, amygdala, hippocampus and orbitofrontal cortex. These regions are also responsible for memory and changes are apparent from the earlies stages of the disease. Indian ink may be most influenced by these neurodegenerative changes among the 12 odors.

In contrast with the previous report using $\mathrm{OE}$ for patients with advanced PD [19], patients with PD in the present study tended to select wrong alternatives instead of "undetectable" as the wrong answers. All the patients with PD selected $70 \%$ or more of wrong alternatives instead of "undetectable" as wrong answers, suggesting the impairment of odor identification and discrimination abilities in the patients with PD. Cognitive impairment is one of the possible explanations of this finding. However, MMSE scores of most of the patients with PD were favorable and no significant statistical correlation was observed between MMSE scores and OE scores in the present study, while MMSE was not conducted in the patients with PVOD. Thus, partial impairment of recognition and discrimination of odor due to neurodegenerative changes in olfactory bulb and upper olfactory cortexes is likely to be responsible for wrong alternatives in patients with PD.

In the present study, we did not evaluate the olfaction and duration from the onset of PD. However, it has been reported that hyposmia is already present bilaterally at the diagnosis of PD, and severity of hyposmia remains essentially constant throughout the course of PD. Hyposmia progression is largely complete before the onset of motor symptoms [17]. On the other hand, PD patients with severe hyposmia has been reported more likely to suffer from PD-specific cognitive dysfunction, such as memory disorders and visuospatial dysfunction [17]. Although no significant correlation was observed between MMSE scores and number of correct answers of $\mathrm{OE}$ in the present study, longterm follow-up of the patients with PD of the present study should be required for early diagnosis of cognitive impairment.

Limitations of the present study was the small number of patients and sex-ratio bias between PD and PVOD groups since olfactory thresholds have been found to decline with age, although this effect is slightly less dramatic in women [17]. Another limitation of this study is the lack of comparison between PD and other neurodegenerative diseases, such as Alzheimer disease. Multi-institutional study including large number of patients with Alzheimer disease as well as PD should be performed for further evaluation of the usefulness of olfactory identification test for screening PD in the patients with olfactory disturbance.

Present results suggested discrepancy between VAS and OE scores, wrong answer in Indian ink and high selected rates of wrong alternatives other than "undetectable" in OE might be useful for differential diagnosis of PD among the elderly with olfactory disturbance.

\section{CONFLICT OF INTEREST}

No potential conflict of interest relevant to this article was reported.

\section{ORCID}

Hisami Fujio https://orcid.org/0000-0001-7766-0523

Ken-ichi Nibu https://orcid.org/0000-0002-5461-4871

\section{REFERENCES}

1. Braak H, DelTredici K, Rub U, deVos RA, Jansen Steur EN, Braak E. Staging of brain pathology related to sporadic Parkinson's disease. Neurobiol Aging. 2003 Mar-Apr;24(2):197-211.

2. Ansari KA, Johnson A. Olfactory function in patients with Parkinson's disease. J Chronic Dis. 1975 Oct;28(9):493-7.

3. Hawkes CH, Shephard BC, Daniel SE. Olfactory dysfunction in Parkinson's disease. J Neurol Neurosurg Psychiatry. 1997 May;62(5): 
436-46.

4. Doty RL. Olfaction in Parkinson's disease. Parkinsonism Relat Disord. 2007;13 Suppl 3:S225-8.

5. Ross GW, Petrovitch H, Abbott RD, Tanner CM, Popper J, Masaki K, et al. Association of olfactory dysfunction with risk for future Parkinson's disease. Ann Neurol. 2008 Feb;63(2):167-73.

6. Baba T, Kikuchi A, Hirayama K, Nishio Y, Hosokai Y, Kanno S, et al. Severe olfactory dysfunction is a prodromal symptom of dementia associated with Parkinson's disease: a 3 year longitudinal study. Brain. 2012 Jan;135(Pt 1):161-9.

7. Doty RL, Shaman P,Applebaum SL, Giberson R, Siksorski L, Rosenberg L. Smell identification ability: changes with age. Science. 1984 Dec;226(4681):1441-3.

8. Doty RL, Shaman P, Kimmelman CP, Dann MS. University of Pennsylvania smell identification test: a rapid quantitative olfactory function test for the clinic. Laryngoscope. 1984 Feb;94(2 Pt 1):176-8.

9. Doty RL, Marcus A, Lee WW. Development of the 12-item CrossCultural Smell Identification Test (CC-SIT). Laryngoscope. 1996 Mar; 106(3 Pt 1):353-6.

10. Kobal G, Hummel T, Sekinger B, Barz S, Roscher S, Wolf S. "Sniffin' sticks": screening of olfactory performance. Rhinology. 1996 Dec; 34(4):222-6.

11. Fujio H, Doi K, Hasegawa S, Kobayakawa T, Nibu K. Evaluation of card-type odor identification test for Japanese patients with olfactory disturbance. Ann Otol Rhinol Laryngol. 2012 Jun;121(6):413-8.

12. McCormack HM, Horne DJ, Sheather S. Clinical applications of vi- sual analogue scales: a critical review. Psychol Med. 1988 Nov;18(4): 1007-19.

13. Ikeda K, Tabata K, Oshima T, Nishikawa H, Hidaka H, Takasaka T. Unilateral examination of olfactory threshold using the Jet Stream Olfactometer.Auris Nasus Larynx. 1999 Oct;26(4):435-9.

14. Mesholam RI, Moberg PJ, Mahr RN, Doty RL. Olfaction in neurodegenerative disease: a meta-analysis of olfactory functioning in Alzheimer's and Parkinson's diseases. Arch Neurol. 1998 Jan;55(1):84-90.

15. Henkin RI, Larson AL, Powell RD. Hypogeusia, dysgeusia, hyposmia, and dysosmia following influenza-like infection. Ann Otol Rhinol Laryngol. 1975 Sep-Oct;84(5 Pt 1):672-82.

16. Welge-Lussen A, Wolfensberger M. Olfactory disorders following upper respiratory tract infections. Adv Otorhinolaryngol. 2006;63:12532.

17. Takeda A, Baba T, Kikuchi A, Hasegawa T, Sugeno N, Konno M, et al. Olfactory dysfunction and dementia in Parkinson's disease. J Parkinsons Dis. 2014;4(2):181-7.

18. Fujio H, Inokuchi G, Fukuda Y, Kuroki S, Kokan N, Nibu K. A new screening tool, an odor identification test card to detect age-related changes in olfaction. Nihon Jibiinkoka Gakkai Kaiho. 2018:121:3843.

19. Watanabe Y, Suzuki K, Miyamoto T, Miyamoto M, Numao A, Fujita $\mathrm{H}$, et al. A card-type odor identification test for Japanese patients with Parkinson's disease and related disorders. Intern Med. 2017 Nov;56(21):2871-8. 